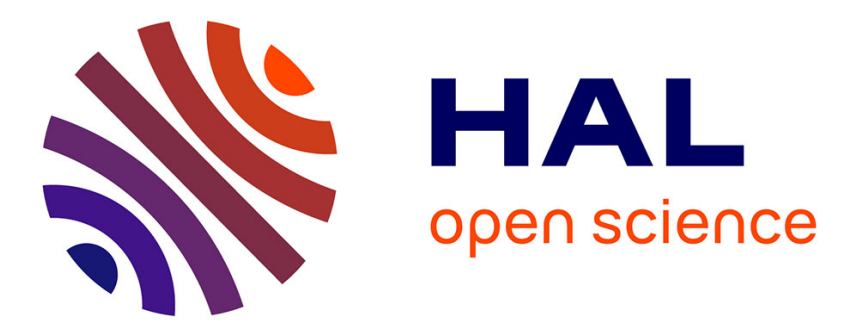

\title{
A Context-Aware Broadcast Protocol for Mobile Wireless Networks
}

Luc Hogie, Gregoire Danoy, Pascal Bouvry, Frédéric Guinand

\section{To cite this version:}

Luc Hogie, Gregoire Danoy, Pascal Bouvry, Frédéric Guinand. A Context-Aware Broadcast Protocol for Mobile Wireless Networks. Modelling, Computation and Optimization in Information Systems and Management Sciences Second International Conference MCO 2008, Sep 2008, Metz, France. pp.507519, 10.1007/978-3-540-87477-5_54. hal-00455546

\section{HAL Id: hal-00455546 https://hal.science/hal-00455546}

Submitted on 10 Feb 2010

HAL is a multi-disciplinary open access archive for the deposit and dissemination of scientific research documents, whether they are published or not. The documents may come from teaching and research institutions in France or abroad, or from public or private research centers.
L'archive ouverte pluridisciplinaire HAL, est destinée au dépôt et à la diffusion de documents scientifiques de niveau recherche, publiés ou non, émanant des établissements d'enseignement et de recherche français ou étrangers, des laboratoires publics ou privés. 


\title{
A Context-Aware Broadcast Protocol for Mobile Wireless Networks
}

\author{
Luc Hogie $^{1}$, Grégoire Danoy ${ }^{2}$, Pascal Bouvry ${ }^{2}$, and Frédéric Guinand ${ }^{3}$ \\ 1 MASCOTTE, joint project CNRS-INRIA-UNSA ${ }^{\star \star}$ \\ 2004, route des lucioles - BP 93 \\ FR-06902 Sophia Antipolis Cedex \\ luc.hogie@sophia.inria.fr \\ 2 University of Luxembourg \\ 6, rue R. Coudenhove-Kalergi \\ L-1359 Luxembourg \\ gregoire.danoy@uni.lu \\ pascal.bouvry@uni.lu \\ 3 University of Le Havre \\ 25, rue Philippe Lebon, BP 1123 \\ FR-76063 Le Havre Cedex \\ frederic.guinand@univ-lehavre.fr
}

\begin{abstract}
Delay Tolerant Networks (DTNs) are a sub-class of mobile ad hoc networks (MANETs). They are mobile wireless networks that feature inherent connection disruption. In particular such networks are generally non-connected. In this paper we focus on defining a broadcast service which operate on DTNs. A number of protocols solving the problem of broadcasting across DTNs have been proposed in the past, but all of them exhibit a static behavior, i.e. they provide no control parameter. However, at the application level, flexible broadcasting schemes are desirable. In particular, it is important that the user (the source of the broadcast message) can control the way the message gets spread across the network. This paper introduces a new broadcasting protocol dedicated to DTNs, called Context-Aware Broadcasting Protocol (CABP), which adapts its greediness according to the "urgency" (priority) of the broadcast message. A formal presentation of its strategy is proposed and through preliminary experiments, the cost-effectiveness of CABP is enlightened.
\end{abstract}

\section{Introduction}

Mobile Ad hoc NETworks (MANETs) are wireless networks composed of nodes able to spontaneously interconnect with other nodes in their geographical neighborhood. Communication does not require any networking infrastructure since, in these networks, nodes communicate directly with each other through the radio medium. To do so, they rely on wireless networking technologies like IEEE802.11a/b/g/n (Wi-Fi) [1] or, to a lesser extent, Bluetooth [2]. When using Wi-Fi, nodes can communicate with other nodes up to a few hundred meters away, in the best case (i.e. when they use Wi-Fi in an environment free of obstacles to the propagation of radio waves).

MANETs are challenging networks mainly because of node mobility. Indeed, node mobility causes fluctuations of the network topology (which result, from the point of view of network nodes, in connection disruptions), as well as variations of the quality of the network links. In particular, unless specific conditions are met (even node distribution, high node density, non-standard radio signal power, etc) the network is very likely to be partitioned. When considering these challenges, mobile ad hoc networks can be referred to as Delay Tolerant Networks (DTNs), as they will in the rest of this paper.

DTNs have a variety of deployments, including Vehicular Ad hoc NETworks (VANETs) [3], sensor networks [4], military networks, etc.

This paper tackles the problem of broadcasting data across DTNs. Put in simple words, broadcasting is the process of sending one message from one node to all other nodes in the network. It has been extensively studied in the past and many broadcast protocols dedicated to mobile ad hoc networks have been proposed.

$\star \star$ Partially supported by the European FET project AEOLUS. 
Static approaches like SBA, Multipoint-Relaying [5] provide efficient solutions. Furthermore, approaches originating from distributed computing and complex systems [6] [7] were described. A recent approach, called MCB, dynamically adapts the broadcast strategy according to user-defined criteria [8]. Although it does not specifically consider preserving the network bandwidth, MCB shares some of its design objectives with the protocol presented in this paper. Unfortunately most of these protocols were designed to operate on MANETs and, because of the stronger constraints inherent to delay tolerant networking, they fail to operate in the latter context. As a consequence new protocols have to be developed for the challenging environment proposed by DTNs.

In the specific context of DTNs, the mere definition of broadcasting has to be revisited. Indeed in a DTN one cannot ensure that all nodes will be reachable. Therefore some studies tackle the broadcasting issue in a different manner. In particular, Alba and al. [9] define the message broadcasting problem as a multi-objective one consisting of:

- maximizing the number of nodes reached;

- minimizing the duration of the process;

- minimizing the bandwidth utilized.

The work presented in this article considers an extension of this definition which introduces the key notion of "message urgency". This new parameter will directly influence the number of nodes reached, the duration of the broadcast process, and the utilization of the network bandwidth. Basically, the more urgent a message is, the greater number of nodes should be reached, the faster possible; and the lesser attention should being paid on bandwidth utilization. We call this broadcast protocol based on message urgency the "context-aware broadcast protocol" (CABP), where the urgency of the broadcast message is viewed as a context information.

The document is organized as follows. Section 2 presents the problem and the CABP protocol. Next in Section 3 the cost-effectiveness of the protocol is analyzed through simulation. Finally Section 4 concludes and presents further research directions.

\section{Description of the protocol}

This section describes the Context-Aware Broadcast Protocol (CABP) by first indicating its objectives, then by detailing the strategy that it relies on, and finally by illustrating its effectiveness through simulation.

\subsection{Objectives}

The design objectives of CABP are threefold:

- It must operate on DTNs, given all the challenges they involve;

- it must provide the user with the ability to control its behavior, for each message processed;

- it must require little information on the network topology.

These three points are detailed in this section.

A broadcast protocol which operates on DTNs. Upon years, a fair wealth of broadcast schemes and protocols have been proposed. Most of them were designed to operate on MANETs. These protocols turn out to be inoperable on DTNs. This has motivated the development of protocols which make use of node mobility to propagate the message, such as AHBP-EX [10,11], DFCN [12] and, more generally, to epidemic broadcast schemes.

A broadcast protocol which is parameterizable. Broadcast protocols most often are targeted to providing low-level network services. In particular, broadcasting is useful in the context of routing [13] [14]. In this context, there is no need to control the behavior of broadcast protocol. This behavior is defined at the design time and cannot be altered afterwards. When looking at broadcast services from the applicative point of view, controllability turns out to be a desirable property. As an example, let us consider an industrial city surrounded by hazardous companies. In order to ensure a certain degree of safety to the population, companies have the possibility to broadcast messages across the available ad hoc networks. In the case of the formation of a toxic cloud caused by one of these companies, it is crucial that a highest-priority message is created and broadcasted across the networks, and that the propagation of this message is not slowed down by advertising messages (or more generally messages of a lower importance), or by cautious network policies whose objective is to control the usage of the bandwidth. 
A broadcast protocol which require only one hop of neighborhood information. Except from Simple Flooding (a node that receives a broadcast message will forward it one single time), broadcast protocols require some form of neighborhood knowledge in order to operate. This knowledge can take the shape of Do I have any neighbors? or Which are my neighbors? or How far is my closest neighbor?, etc. Wu and Lou [15] have defined a classification which takes into account this amount of neighborhood knowledge that is required. They roughly define two classes: centralized and localized protocols. On the one hand, centralized protocols require global network information. Since global network information is inherently not achievable in DTNs, centralized protocols are not suitable to broadcasting in those networks. On the other hand, localized protocols require local neighborhood information, that is information on the network topology in the first and/or second hop around the node that is executing the protocol. Protocols like AHBP-EX [16] and SBA [17] use 2-hops of neighborhood information. They exhibit the most effective strategies. However in DTNs, because of the potentially very dynamic nature of the network, 2-hops of neighborhood information may be not be achievable. In the context of DTNs, broadcasting protocols which require only 1-hop of neighborhood information are highly desirable. This is for example the case of Flooding with Self Pruning. DFCN [12] [18] and CABP are also designed in this way.

A number of broadcast protocols already meet the aforementioned design objectives; that is they operate on DTNs, they provide control on the way they behave and they require 1-hop neighborhood information. Such protocols include probabilistic schemes, distance and area-based methods [11]. Unfortunately there does not exist guidelines on how to set their parameters in order to obtain the desired effect, if possible. For example, the probabilistic scheme (nodes forwarded according to a probability defined by the user) cannot be applied in the case of the propagation of low urgency messages: experimentation showed that probabilities below 0.5 cannot be applied. As a matter of fact, metrics like network coverage or bandwidth utilization do not obey to linear functions of basic parameters such as broadcast probabilities. Recent studies [8] propose a way of parameterizing the broadcast process so as it will target to certain objectives. However, contrarily to what is presented hereinafter, these objectives do not consider the minimization of the network bandwidth.

\subsection{Requirements}

In order to operate, CABP requires that the nodes must:

- know the IDs of their neighbor nodes;

- locally maintain a set of node IDs associated to every message they receive;

Additionally message headers must contain:

- the ID of the node which sent the message, referred to as the source node (note that the source node is not the node which initiates the broadcast process, it is the one which forwards the message);

- the list of IDs of the neighbors of their source node (plus one additional word indicating the end of the neighbor list);

- one byte coding the urgency of the message;

- three bytes ${ }^{4}$ coding the number of seconds before the message expires.

The message header should hence be structured as shown on Figure 1. Using 8 bits for encoding message urgency should provide appropriate precision in the context of this paper.

\subsection{Mathematical model}

In the following, we will assume that given a node $n$, The ID of $n$ 's neighbors is noted $N(n)$, and given a message $m$, the source node of $m$ is given by $s(m)$. Additionally, the urgency of a message $m$ is given by $u(m)$. It is defined in [0..1]. The greater value for $u(m)$, the greater urgency for $m$.

${ }^{4}$ Three bytes should satisfy the majority of the possible applications since it makes it possible to keeps messages almost 200 days. 


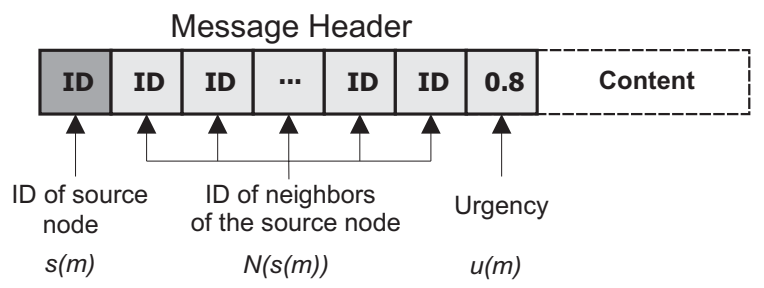

Fig. 1. The CABP messages header.

General principle In order to make it clear the design objectives for CABP, consider the two extreme values of importance: 0 and 1.

At the lower bound, an urgency of 0 does not imply any requirement in terms of the speed and delivery ratio of the message. In this case where the urgency is minimal, a great attention should be paid to utilizing as little resources as possible. In order to save resources, nodes forward messages with a probability that decreases when the number of their neighbors increases. In the case of 0 -urgency, a node $n$ forwards the broadcast message with a probability $P(n, m)=\frac{1}{|N(n)|^{\frac{1}{a}}}$, given that the set of known neighbors of a node $n$ is given by $N(n)$. Hence when the neighbor density is high, individual nodes forward messages with a low probability; but the high number of nodes statistically ensures that one node will forward the message. $a$ is used as a calibration value for the protocol. It determines how fast the forward probability decreases. For the sake of simplicity, in the following we will consider that this probability decreases in a linear fashion, that is $a=1$.

At the upper bound, an urgency of 1 means that the message should be broadcasted at any cost, as fast as possible, and in such a manner that the delivery ratio is $100 \%$. In that case, the resources available are utilized regardless of their utilization by other applications - which are considered of a lesser urgency. Then nodes forward the broadcast message with a probability of 1 . In this extreme case, the number of neighbors is not taken into account.

Behavioral requirements Formally speaking, the probability $P(n, m)$ that a node $n$ forwards a broadcast message $m$ depends both on:

- the urgency $u(m)$ of the message $m$;

- the number $|N(n)|$ of neighbors of the broadcasting node $n$. $|N(n)|$ is defined in [1.. $+\infty]$. It is not defined

below 1 because if a node $n$ has less than one neighbor, it does not even consider forwarding messages.

$P(n, m)$ must satisfy two requirements, as defined in the following.

On the one hand, by looking at the extreme urgency values 0 and 1 as described hereinbefore, it comes that $P(m, n)$ must exhibit the following properties at the limits, as they are defined in the previous section:

$$
\left\{\begin{array}{l}
\lim _{u(m) \rightarrow 0} \mathrm{P}(\mathrm{m}, \mathrm{n})=\frac{1}{|N(n)|^{\frac{1}{a}}} \\
\lim _{u(m) \rightarrow 1} \mathrm{P}(\mathrm{m}, \mathrm{n})=1
\end{array}\right.
$$

On the other hand, it is desirable that $P(m, n)$ is continuous and that altering $u(m)$ impacts the behavior of the protocol in a linear manner. Indeed the behavior of the protocol is parameterized by the value of $u(m)$, whose the value is intended to be defined by a human operator. Ensuring a linear change of behavior of $P(m, n)$ when $u(m)$ varies is the best way the allow the human operator to have good control of the "urgency knob". In mathematical words, $P(m, n)$ must be a linear function of $u(m)$. That is there must exist two functions $f(n)$ and $g(n)$ so that $P(m, n)=f(n) \times u(m)+g(n)$ :

Proposed model The most straightforward mathematical expression which meets the aforementioned requirements defines that the probability $P(n, m)$ that a node $n$ forwards a message $m$ is:

$$
P(n, m)=\frac{1-u(m)}{|N(n)|^{\frac{1}{a}}}+u(m)
$$

Which can be put in the form $f(x)=x a+(x-1) b$, allowing $f(x)$ to morph from $a$ to $b$, depending on $x$. 


\subsection{Triggers}

The mathematical model described in section 2.3 is applicable in two different situations. First when a node $n$ receives a message $m$ from one of its neighbors, it will forward it according to a probability $P(n, m)$. Second, when a node $n$ discovers a new neighbor, it considers forwarding every message it is currently carrying. This forward happens with the same probability $P(n, m)$.

\subsection{Random Assessment Delay}

Most often broadcast protocols make use of a Random Assessment Delay (commonly referred to as the RAD), which allows nodes to "wait before send". More precisely, when a node receives a broadcast message and immediately decides to forward it, it does not radio-transmit at once. Instead it will wait a random amount of time. This prevents nodes that receive simultaneously the same message from a common neighbor to forward it at the same moment. A simultaneous collective re-emission would result in a high risk of packet collision.

Broadcast protocols use a generic method for determining the assessment delay. This method consists in picking up a random number in [0, max_delay]. CABP propose an extension of this strategy by benefit from nodes' neighborhood knowledge. Formally speaking, when a node $n$ receives a message $m$ from a source node $s(m), n$ computes an assessment delay on the basis of the neighborhood of $s(m)$. As detailed in Section 2.2, messages embed (in their header) the ordered list of neighbors' ID $N \circ s(m)$ of their source node $s(m)$. On reception of a message $m$, node $n$ determines the offset $o(n, m)$ of its own ID in the list of node ID embedded in $m$. The assessment delay that $n$ will wait before

$$
d(n, m)=q \times o(n, m)
$$

In this equation $q$ "slices" the time, meaning that the forward of a message happens only after a delay of $n \times q$ seconds, where $n \in \mathbb{N}$. We suggest $q=0.1 s$. Note that the determination of the delay does not depend on the urgency of the message. One may think that urgent messages should be forwarded with lower delays, but doing this would increase the risk of packet collision and would finally lead to harmfully lower delivery ratio.

This technique for the determination of the assessment delay ensures a number of properties. First, if the transmission of a message lasts less than $q$ seconds, no collision occurs. Second, the sparser is the network, the faster the message gets disseminated. In the extreme case (if no competition for the medium happens no risk of collision exists) the message is forwarded with no delay.

\subsection{Node memory}

CABP makes use of a generic technique which consists in maintaining a node-local history of the others nodes' IDs which are known to have received a given message. Basically a node remembers the nodes to which it sent the message in the past. In the same manner, it remembers the neighbors of the node which communicated him the message, since they also received it. This general technique can be applied only when 1-hop neighborhood information is available. It proves an effective way to reduce the number of transmission of broadcast messages. The technique requires that nodes individually manage an associative map

$$
i d_{\text {msg }} \rightarrow\left\{i d_{\text {node }_{1}}, i d_{\text {node }_{2}}, \ldots, i d_{\text {node }_{n}}\right\}
$$

which establishes a one-to- $n$ relation from one message ID to a set of node IDs. This table is updated in the case of message emissions and receptions.

On the one hand, just before a node $n$ emits a message $m$, it builds a set $N(n)$ consisting of the ID of its neighbors. These neighbors are considered to be actual recipients of the message. Then node $n$ associates $N(n)$ to the ID of the message $m$, by storing the relation $m \rightarrow N(n)$ in its local associative table. Also the IDs in $N(n)$ are embedded into the message header.

On the other hand, on reception of a message $m$, a node $n_{2}$ obtains a list $N(s(m))$ of the neighbors of its source node $s(m)$, as well as the ID of $s(m)$. Then node $n_{2}$ stores the relation

$$
m \rightarrow N \circ s(m) \cup\{s(m)\}
$$

into its local associative table. 
The knowledge provided by the associative table is used when a node considers forwarding a message. Before transmitting, it tests if there exists at least one of its neighbors whose the ID is not yet stored in the set associated to the message's ID. If one (or more) of such neighbor is found, the message is forwarded.

The lifetime of the set of IDs for a given message is the same as the lifetime of the message. As a consequence, when a message expires, all the local sets associated to it are erased from the memory of all nodes.

\section{Experimentation}

The behavior of CABP is investigated through simulation. This section first describes the tools that we use as well the conditions under which CABP was tested. Then preliminary results are presented.

\subsection{Simulation environment}

CABP was prototyped and studied using the Madhoc wireless network simulator. Madhoc was initially targeted at the design and experimentation of broadcasting protocols. As such, it provides a framework that is suited to their development, and it comes with a set of tools that simplifies the monitoring of such highly distributed applications. In addition to that, it offers a set of mobility models allowing the simulation of a variety of environmental conditions. ${ }^{5}$.

Our simulation campaign relied on the following parameters. The network is composed of 500 nodes evolving in a bounded squared area of $1 \mathrm{~km}^{2}$. The nodes mobility obeys to the rules defined by the Human Mobility Model [19]. Briefly, the Human Mobility Model defines that the simulated area exhibits a set of spots. A spot is a circular area surrounded by a wall (walls constitute obstacles to the propagation of radio waves). Within a spot, the nodes move in random directions. When a node gets out of a spot, it chooses the closest spot that it has not yet visited. Thus every node maintains a local history of the spots they visit. Once all spots have been visited, the local history is cleared.

Although all nodes move independently from one another, the human mobility model permits the emergence of mobility patterns such as temporary group mobility, lines and clusters of nodes. The human mobility model was chosen because of its ability to reproduce such phenomenons.

The network environment we considered consists of 50 spots evenly located across the simulation area. The distance between spots is constrained so as it cannot be lower than $50 \mathrm{~m}$. Each spot has a radius randomly chosen between 20 and 30 meters. The graph of the initial network is represented in Figure 3. The simulation considers the broadcast of one single message, from one node to as many destination nodes as possible. Note that the initiator node is chosen so that it is in the middle of the longest path in the network graph.

\begin{tabular}{|l|c|}
\hline number of nodes & 500 \\
\hline number of spots & 50 \\
\hline mininum dist between spots & $50 \mathrm{~m}$ \\
\hline spot radius & randomly chosen in $[20,30] \mathrm{m}$ \\
\hline simulation area surface & $1 \mathrm{~km}^{2}$ \\
\hline simulation area shape & square \\
\hline message urgency & $\{0,0.3,0.6,1\}$ \\
\hline
\end{tabular}

Fig. 2. The parameters used for the experimentation campaign.

\subsection{Results}

In order to illustrate the behavior of the CABP protocol. We will consider the following metrics:

\footnotetext{
${ }^{5}$ The source code of the Madhoc simulator is available at the following web address: http://agamemnon.uni.lu/ $\sim \operatorname{lhogie} /$ madhoc/
} 


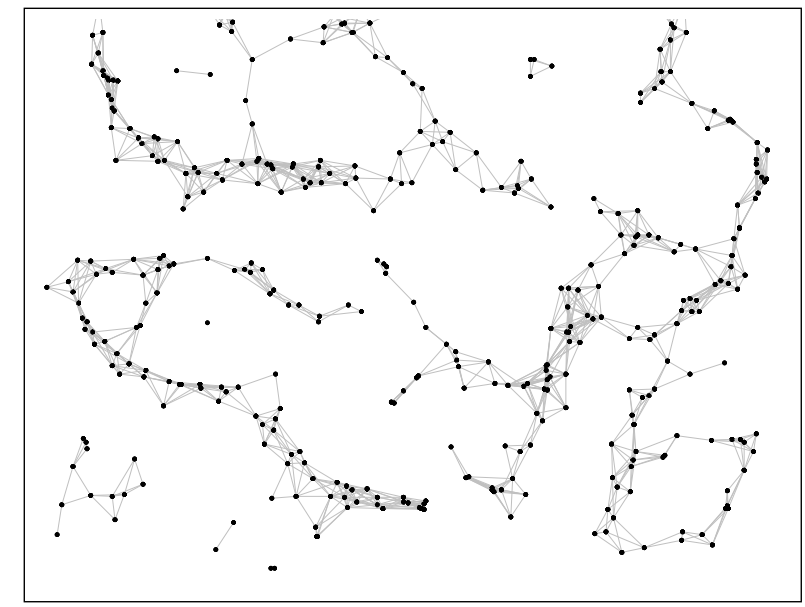

Fig. 3. The mobility model which rules the dynamics of the network defines a number of center of interests (called spots) where the nodes go to and stay for a while. A few parameters controlling the mobility permits to define several realistic scenarios of human mobility.

- the evolution of the coverage upon time;

- the number of message emissions upon time (this reflects the utilization of the bandwidth);

- the number of emissions carried out for reaching a given coverage;

- the evolution of the memory requirements upon time.

Bandwidth utilization/time The number of emissions is an important measure because it has a direct impact on the network bandwidth which is used along the broadcasting process. The number of emissions has to be kept as low as possible, taking into account the importance of the message: the most important it is, the less care should be taken to the number of emissions. Figure 4 shows that a high urgency leads to numerous emissions, but also that it has the desirable effect to broadcast the message fast. However when reducing the importance of the message, the number of emissions dramatically lessens. This result indicates that the importance of a message should be carefully chosen. Setting a too high importance leads to a high bandwidth utilization, while setting too low importance slightly delays the message, still ensuring a complete dissemination of the message.

Delivery ratio/time Figure 5 shows the evolution of the delivery ratio upon time. The delivery ratio is the ratio of the nodes which has received the message. The simulation process is considered terminated as soon as a delivery ratio reaches a value of 1 (the message has been delivered to every node). What counts is the time required to reach a delivery ratio of 1 . The more important a message is, the faster a delivery ratio of 1 should be reached. Figure 5 shows that when the importance of the message is 1 , a high delivery ratio is reached fast. It also shows that this velocity of the broadcast process is not exactly proportional to the importance of the message. This attests that the probability function has room for improvement.

Note that there is no guarantee that the broadcasting process will reach every nodes. Theoretically the probability that a given node never meets another node which has received the message is not null, although insignificant.

Local memory utilization Figure 6 shows that when broadcasting in a network composed of 500 nodes moving in a 1 square kilometer area, the memory size required to store the local history for one message is significantly less than $1 \mathrm{~Kb}$. This value assumes that the ID of the nodes is stored on 6 bytes, as it is the case when using MAC or IPv6 addresses as nodes ID. Even if all nodes got in contact with all other nodes, they would have to store $500 \mathrm{IDs}$, which would require $3 \mathrm{~Kb}$ of memory. 


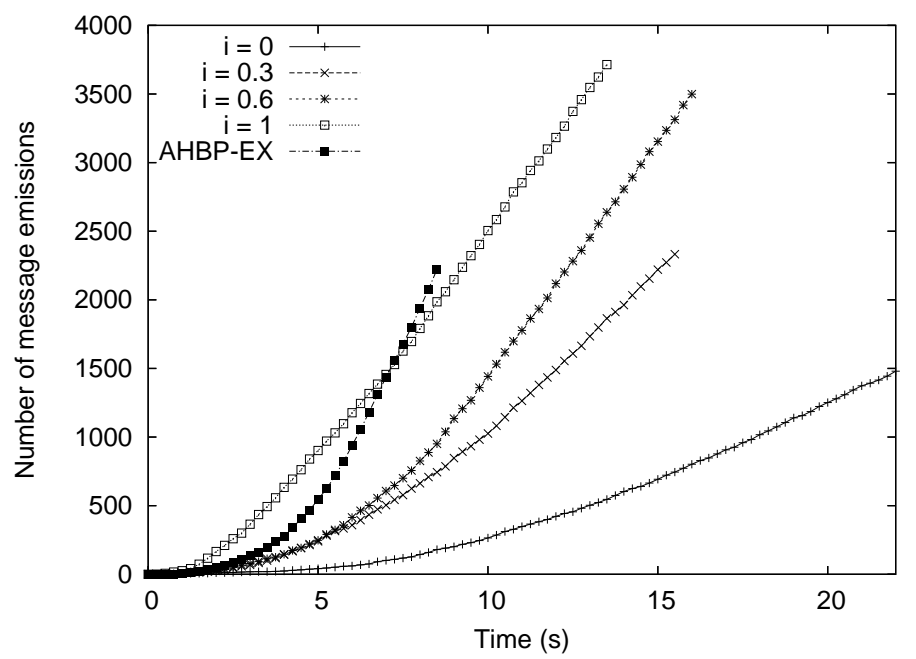

Fig. 4. If message urgency does not have such a great impact on the time required for message dissemination (makespan), it does seriously impact the bandwidth utilization. As illustrated here low-urgency messages require significantly less bandwidth to get disseminated.

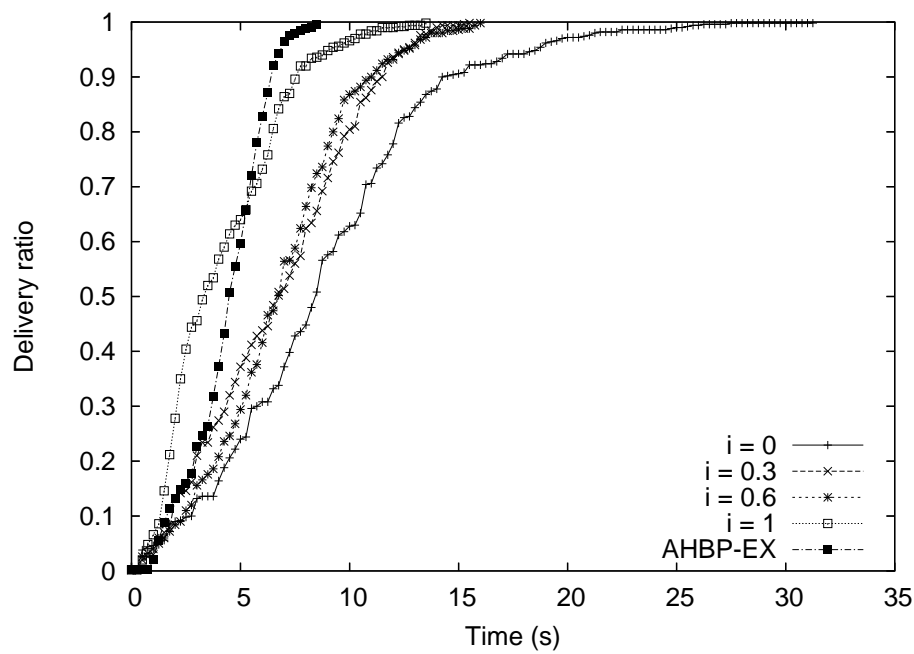

Fig. 5. The evolution of the delivery ratio depends on the message urgency. Less important messages are broadcasted using a smooth strategy whose aim is to use little network resources. A consequence is that their complete dissemination takes longer. 


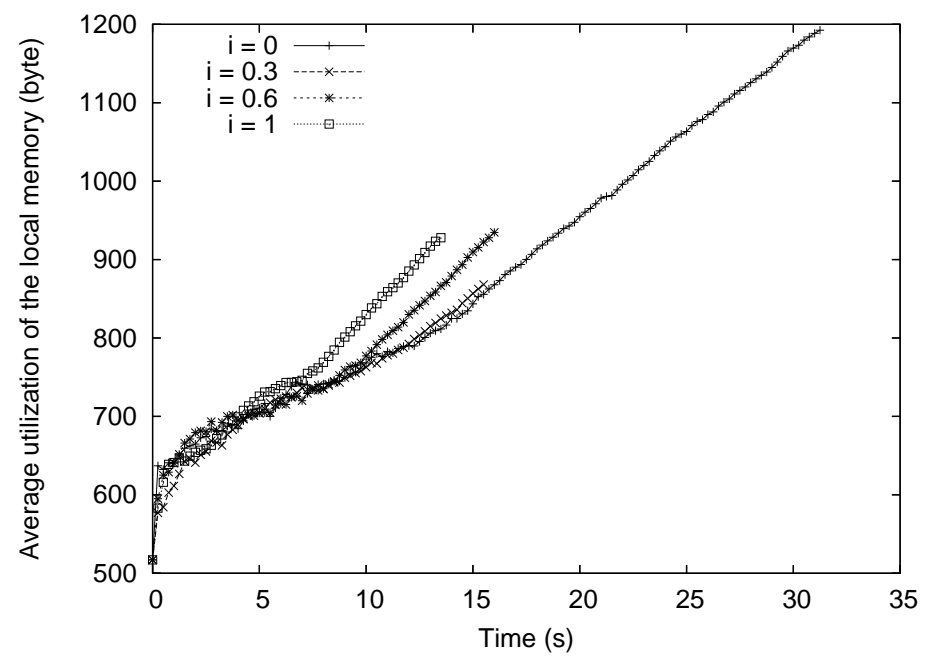

Fig. 6. The utilization of memory local to every node directly depends on the velocity of the broadcasting process. This figure illustrates the fact that the way nodes forward the broadcast messages that they hold when they meet new neighbors depends on message urgency.

\section{Conclusion and future works}

This paper introduced the Context-Aware Broadcasting Protocol (CABP). Unlike most existing broadcast protocols, CABP is to provides a parameterizable broadcasting protocol for Mobile Ad hoc NETworks (MANETs) and Delay Tolerant Networks (DTNs).

We experimentally demonstrated that the "urgency" parameter of CABP provides the desired behavior. Indeed, the less urgent is the message, the less resources are utilized in terms of bandwidth and memory usage. On the contrary, the more urgency the message has, the quicker the broadcast process is, regardless of the resource utilized to perform it.

In addition to this, CABP proceeds regardless of the network density, which make it usable in any network condition, and in particular it can use employed in the specific context of the DTNs.

Further works include the refinement of the probabilistic model for the protocol, so that its behavior will be more linear, i.e. more controllable by the user.

\section{References}

1. ANSI/IEEE: Ansi/ieee std 802.11, 1999 edition (r2003). wireless lan medium access control (mac) and physical layer (phy) specifications (1999)

2. the Bluetooth SIG, I.: Specification of the bluetooth system, core, volume 1, version 1.1

3. Torrent-Moreno, M., Jiang, D., Hartenstein, H.: Broadcast reception rates and effects of priority access in 802.11based vehicular ad-hoc networks. In: Vehicular Ad Hoc Networks. (2004) 10-18

4. Akyildiz, I., Su, W., Sankarasubramaniam, Y., Cayirci, E.: A survey on sensor networks. IEEE Communications Magazine 8 (August 2002) 102-114

5. Stojmenovic, I., Wu, J.: Broadcasting and activity scheduling in ad hoc networks. S. Basagni, M. Conti, S. Giordano, I. Stojmenovic, Eds., Mobile Ad Hoc Networking (2004) 205-229

6. Colagrosso, M.D.: Intelligent broadcasting inmobile ad hoc networks: three classes of adaptive protocols. EURASIP J. Wirel. Commun. Netw. 2007(1) (2007) 25-25

7. Khelil, A.: Contact-based buffering for delay-tolerant ad hoc broadcasting. Comput. Commun. 30(16) (2007) 3144-3153

8. Barritt, B., Malakooti, B., Guo, Z.: Intelligent multiple-criteria broadcasting in mobile ad-hoc networks. Icn 0 (2006) 761-768 
9. Alba, E., Dorronsoro, B., Luna, F., Nebro, A.J., Bouvry, P., Hogie, L.: A cellular multi-objective genetic algorithm for optimal broadcasting strategy in metropolitan manets. Comput. Commun. 30(4) (2007) 685-697

10. Peng, W., Lu, X.: Ahbp: An efficient broadcast protocol for mobile ad hoc networks. J. Comput. Sci. Technol. 16(2) (2001) 114-125

11. Williams, B., Camp, T.: Comparison of broadcasting techniques for mobile ad hoc networks. In: Proc. of the ACM International Symposium on Mobile Ad Hoc Networking and Computing (MOBIHOC). (2002) 194-205

12. L. Hogie, P. Bouvry, F.G.G.D.E.A.: A Bandwidth-Efficient Broadcasting Protocol for Mobile Multi-hop Ad hoc Networks. In: Demo proceeding of the 5th International Conference on Networking (ICN'06), IEEE (October 2006)

13. Broch, J., Maltz, D.A., Johnson, D.B., Hu, Y.C., Jetcheva, J.: A performance comparison of multi-hop wireless ad hoc network routing protocols. In: MobiCom '98: Proceedings of the 4th annual ACM/IEEE international conference on Mobile computing and networking, New York, NY, USA, ACM Press (1998) 85-97

14. Jones, E.P., Li, L., Ward, P.A.: Practical routing in delay-tolerant networks. In: Proceedings of the 2005 ACM SIGCOMM workshop onDelay-tolerant networking, Philadelphia, PA, USA (August 2005) 237-243

15. Wu, J., Lou, W.: Forward-node-set-based broadcast in clustered mobile ad hoc. Wireless Communications and Mobile Computing 3 (2003) 155-173

16. Peng, W., Lu, X.: Ahbp: An efficient broadcast protocol for mobile ad hoc networks. Journal of Computer Science and Technology 16(2) (2001) 114-125

17. Peng, W., Lu, X.C.: On the reduction of broadcast redundancy in mobile ad hoc networks. In: MobiHoc '00: Proceedings of the 1st ACM international symposium on Mobile ad hoc networking \& computing, IEEE Press (2000) 129-130

18. Hogie, L., Guinand, F., Bouvry, P.: A Heuristic for Efficient Broadcasting in the Metropolitan Ad Hoc Network. In: 8th Int. Conf. on Knowledge-Based Intelligent Information and Engineering Systems (KES'04). (2004) 727-733

19. Hogie, L.: Mobile Ad Hoc Networks: Modelling, Simulation and Broadcast-based Applications. PhD thesis, University of Le Havre, University of Luxembourg (April 2007) 